\title{
THE EXISTENCE OF FLAT COVERS OVER NOETHERIAN RINGS OF FINITE KRULL DIMENSION
}

\author{
JINZHONG XU
}

(Communicated by Wolmer V. Vasconcelos)

\begin{abstract}
Bass characterized the rings $R$ with the property that every left $R$-module has a projective cover. These are the left perfect rings. A ring is left perfect if and only if the class of projective $R$-modules coincides with the class of flat $R$-modules, so the projective covers over these rings are flat covers. This prompts the conjecture that over any ring $R$, every left $R$-module has a flat cover. Known classes of rings for which the conjecture holds include Von Neumann regular rings (trivially), the left perfect rings (Bass), Prufer domains (Enochs), and then more generally, all right coherent rings of finite weak global dimension (Belshoff, Enochs, $\mathrm{Xu}$ ).

In this paper we show that the conjecture holds for all commutative Noetherian rings of finite Krull dimension and so for all local rings and all coordinate rings of affine algebraic varieties.
\end{abstract}

\section{Preliminaries}

We will use the terminology of Enochs [4]. We recall that if $\mathscr{F}$ is a class of left flat $R$-modules, a linear map $\varphi: F \rightarrow M$ with $F \in \mathscr{F}$ is called an $\mathscr{F}$ precover of $M$ if $\operatorname{Hom}_{R}(G, F) \rightarrow \operatorname{Hom}_{R}(G, M) \rightarrow 0$ is exact for all $G \in \mathscr{F}$. If furthermore any linear map $f: F \rightarrow F$ such that $f \varphi=\varphi$ is an automorphism of $F$, then $\varphi: F \rightarrow M$ is called an $\mathscr{F}$-cover. If an $\mathscr{F}$-cover exists, it is unique up to isomorphism. If, for example, $\mathscr{F}$ is the class of flat modules, then an $\mathscr{F}$-precover (or cover) is called a flat precover (or cover). Note that since projective modules are flat, flat precovers are necessarily surjective. $\mathscr{F}$-pre-envelopes and envelopes are defined dually.

We note that in Auslander and Reiten's terminology [1], an $\mathscr{F}$-precover and an $\mathscr{F}$-cover are called a right $\mathscr{F}$-approximation and a minimal right $\mathscr{F}$ approximation respectively. In their language, our main result says that the class of flat modules is contravariantly finite in the category of left $R$-modules with $R$ commutative, Noetherian, and of finite Krull dimension.

A left $R$-module $C$ is called to be cotorsion if $\operatorname{Ext}_{R}^{1}(F, C)=0$ for all flat left modules $F$. If this holds, it follows that $\operatorname{Ext}_{R}^{i}(F, C)=0$ for all flat left modules $F$ and all $i \geq 1$. It also follows easily that if $0 \rightarrow C^{\prime} \rightarrow C \rightarrow C^{\prime \prime} \rightarrow 0$

Received by the editors April 7, 1993.

1991 Mathematics Subject Classification. Primary 13C05, 13C11.

Key words and phrases. Flat covers, Krull dimension, cotorsion modules. 
is exact with $C^{\prime}$ cotorsion, then $C$ is cotorsion if and only if $C^{\prime \prime}$ is cotorsion. If $\varphi: F \rightarrow M$ is a flat cover of $M$, then the $\operatorname{ker}(\varphi)$ is cotorsion [6, Lemma 2.2]. Conversely, if $\varphi: F \rightarrow M$ is a linear map with $F$ flat and $\operatorname{ker}(\varphi)$ cotorsion, then it is a flat precover of $M$. We also know that a module $M$ has a flat cover if it has a flat precover [4, Theorem 3.1].

We discuss flat covers of modules in an exact sequence first. Then, by a result of Raynaud and Gruson [9, Corollary 3.2.7] on a commutative Noetherian ring of finite Krull dimension, we show that over such a ring every module has a flat cover. This holds for all local rings and coordinate rings of affine algebraic varieties.

\section{THE MAIN RESULT}

Lemma 2.1. If $M \subset G$ is a submodule of a left $R$-module $G$ such that $G / M$ is flat and where $G$ has a flat cover, then $M$ has a flat cover.

Proof. Let $\varphi: F \rightarrow G$ be the flat cover of $G$. Then $F / \varphi^{-1}(M) \cong G / M$ is flat, so $\varphi^{-1}(M)$ is flat. Since $F \rightarrow G$ is a flat cover, it is easy to see that $\varphi^{-1}(M) \rightarrow M$ is a flat precover. By Theorem 3.1 of [4], $M$ then has a flat cover.

Theorem 2.2. If $R$ is right coherent, a left $R$-module $M$ has a flat cover if and only if there is an exact sequence

$$
0 \rightarrow M \rightarrow G \rightarrow L \rightarrow 0
$$

such that $L$ is flat and such that $G$ is cotorsion and has a flat cover.

Proof. Given such a sequence $0 \rightarrow M \rightarrow G \rightarrow L \rightarrow 0$, by Lemma 2.1, $M$ has a flat cover.

Now suppose that $M$ has a flat cover $F \rightarrow M$ with kernel $C$. Then $C$ is cotorsion (Enochs [6, Lemma 2.2]). Let $F \rightarrow P$ be a pure injective envelope of $F$ with cokernel $L$. By Proposition 4.1 of [8], $L$ is flat. Then using a pushout of $F \rightarrow M$ and $F \rightarrow P$, we get a commutative diagram

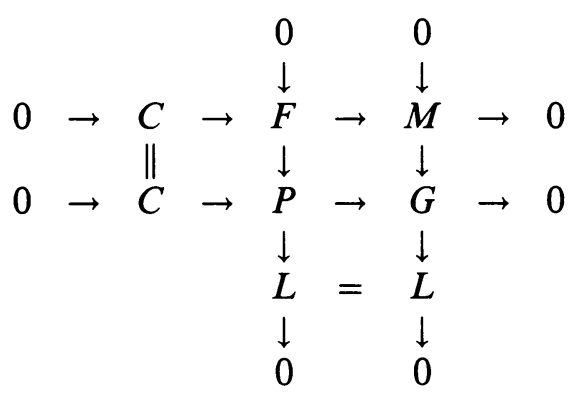

with exact rows and columns. Here $C$ and $P$ are cotorsion, so is $G$. As noted, $L$ is flat and $G$ has a flat cover, so we have the desired exact sequence.

Theorem 2.3. Let $R$ be right coherent and $0 \rightarrow A \rightarrow B \rightarrow C \rightarrow 0$ be an exact sequence of left $R$-modules. If both $A$ and $C$ have flat covers, then $B$ has $a$ flat cover.

Proof. Let $\psi: F \rightarrow C$ be a flat cover of $C$. Consider the pullback diagram of 
$f \rightarrow C$ and $B \rightarrow C:$

$$
\begin{aligned}
& 0 \rightarrow \underset{\|}{A} \rightarrow \underset{\downarrow \pi_{1}}{P} \stackrel{\pi}{\rightarrow} \underset{\downarrow \psi}{F} \rightarrow 0 \\
& 0 \rightarrow A \stackrel{f}{\rightarrow} \quad B \quad \stackrel{g}{\rightarrow} \quad C \quad \rightarrow \quad 0
\end{aligned}
$$

We claim that if $P$ has a flat cover, say $\sigma: G \rightarrow P$, then $\pi_{1} \sigma: G \rightarrow B$ is a flat precover of $B$.

For any linear map $\sigma_{1}: G^{\prime} \rightarrow B$ with $G^{\prime}$ flat, consider the map $g \sigma_{1}: G^{\prime} \rightarrow C$. Since $\psi: F \rightarrow C$ is a flat cover of $C$, there is a linear map $q: G^{\prime} \rightarrow F$ such that $g \sigma_{1}=\psi q$. Namely, we have the following commutative diagram:

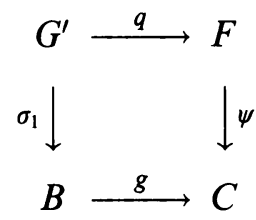

By the property of pullbacks (see [10]), there is a unique linear map $p: G^{\prime} \rightarrow P$ such that $\sigma_{1}=\pi_{1} p, q=\pi p$. Now, we consider the following diagram:

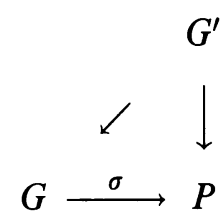

Since $\sigma: G \rightarrow P$ is the flat cover of $P$, there is a linear map $h: G^{\prime} \rightarrow G$ such that $p=\sigma h$. This implies that $\sigma_{1}=\pi_{1} p=\pi_{1} \sigma h$. Therefore, $h$ is our desired map.

In order to show that $P$ has a flat cover, since $A$ has a flat cover, by Theorem 2.2, we can consider the exact sequence

$$
0 \rightarrow A \rightarrow G \rightarrow D \rightarrow 0
$$

where $G$ is cotorsion and has a flat cover and $D$ is flat. Then we have the following diagram:

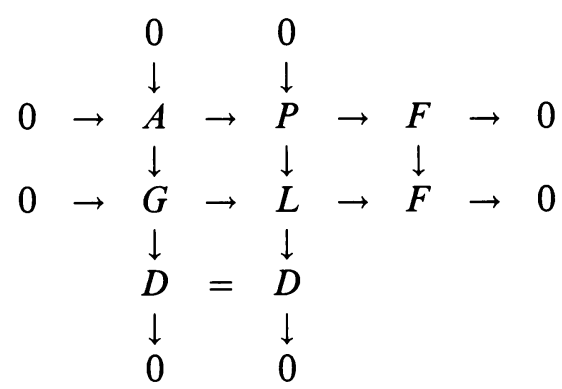

Here, $L$ is the pushout of $A \rightarrow P$ and $A \rightarrow G$. Since $G$ is cotorsion and $F$ is flat, the exact sequence $0 \rightarrow G \rightarrow L \rightarrow F \rightarrow 0$ is split. This implies that $L$ has a flat cover because both $G$ and $F$ have flat covers.

Finally, we consider the exact sequence

$$
0 \rightarrow P \rightarrow L \rightarrow D \rightarrow 0
$$

where $L$ has a flat cover and $D$ is flat. By Lemma 2.2, $P$ has a flat cover. 
Theorem 2.4. Let $R$ be right coherent and $0 \rightarrow A \rightarrow B \rightarrow C \rightarrow 0$ be an exact sequence of left $R$-modules. If both $A$ and $B$ have flat covers, then $C$ also has a flat cover.

Proof. Since $A$ has a flat cover, by Theorem 2.2, we have the exact sequence

$$
0 \rightarrow A \rightarrow G \rightarrow D \rightarrow 0
$$

where $D$ is flat and $G$ is cotorsion and has a flat cover.

Consider the following diagram:

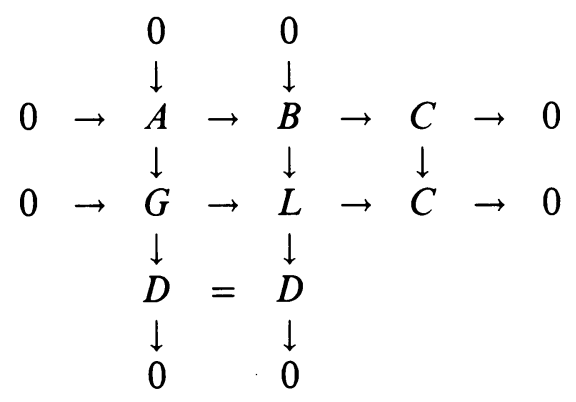

Since both $B$ and $D$ have flat covers, by Theorem $2.3, L$ also has a flat cover. Let $\psi: F \rightarrow L$ be a flat cover of $L$. Consider the following diagram:

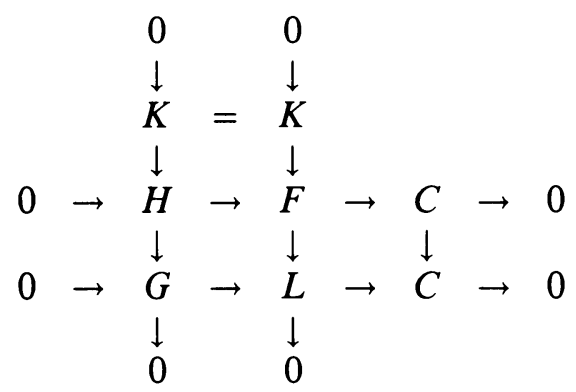

Here, $H$ is a pullback of $F \rightarrow L$ and $G \rightarrow L$. Note that since both $K$ and $G$ are cotorsion, it follows that $H$ is also cotorsion. But, the fact that $H$ is cotorsion and $F$ is flat implies that $F \rightarrow C$ is a flat precover of $C$.

Theorem 2.5. Let $R$ be right coherent. Then the following statements are equivalent.

(a) Any torsion left $R$-module has a flat cover.

(b) For any exact sequence, $0 \rightarrow A \rightarrow B \rightarrow C \rightarrow 0$, whenever both $B$ and $C$ have flat covers, so does $A$.

Proof. (a) $\Rightarrow$ (b) Consider the following diagram:

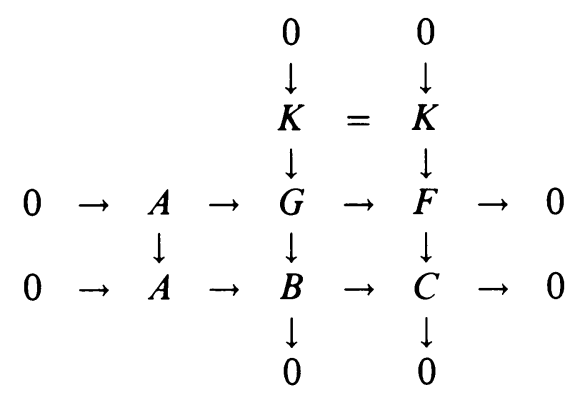


Here, $F \rightarrow C$ is the flat cover of $C$ and $G$ is the pullback of $F \rightarrow C$ and $B \rightarrow C$. We know that since $K$ is cotorsion, by (a), it has a flat cover. Now both $K$ and $B$ have flat covers, so by Theorem 2.3, $G$ also has a flat cover. However, this implies that $A$ has a flat cover because $F$ is flat and $G$ has a flat cover.

(b) $\Rightarrow$ (a) Let $G$ be cotorsion. We try to show that $G$ has a flat cover by using (b). Consider the exact sequence $0 \rightarrow G \rightarrow E \rightarrow L \rightarrow 0$, where $G \rightarrow E$ is the injective envelope of $G$. Then $E$ has a flat cover [6, Proposition 1.1]. Let $F \rightarrow E$ be a flat cover of $E$. Consider the diagram:

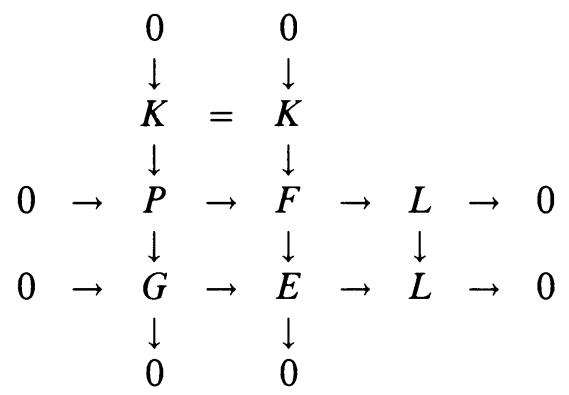

where $P$ is the pullback of $G \rightarrow E$ and $F \rightarrow E$. Then $K$ is cotorsion and it follows that $P$ is also cotorsion. This implies that $F \rightarrow L$ is a flat precover of $L$ and then that $L$ has a flat cover. Now, by the assumption (b), $G$ has a flat cover because both $E$ and $L$ have flat covers.

Note that Enochs proved that every cotorsion module over a commutative Noetherian ring of finite Krull dimension has a flat cover [7, Theorem 3.1]. Therefore, Theorem 2.5 holds for this case. Now we are ready to prove our main result.

Theorem 2.6. If $R$ is a commutative Noetherian ring of finite Krull dimension, then every $R$-module has a flat cover.

Proof. Let the Krull dimension of $R$ be $n$. If $M$ is an $R$-module, let

$$
0 \rightarrow M \rightarrow E^{0} \rightarrow \cdots \rightarrow E^{n-1} \rightarrow C \rightarrow 0
$$

be a partial injective resolution of $M$. Note that for any flat module $F$, proj. $\operatorname{dim}_{R} F \leq n$ (Raynaud and Gruson [9, Corollary 3.2.7]). Then it is easy to see that $C$ is cotorsion. By the previous note, $C$ and all $E^{0}, \ldots, E^{n-1}$ have flat covers. A repeated application of Theorem 2.5 then shows that $M$ has a flat cover.

Corollary 2.7. Let $R$ be commutative Noetherian ring. Then for any $R$-module $M$ and any prime $p \in \operatorname{Spec} R, M_{p}$ has a flat cover as $R$-module.

Proof. For any prime $p \in \operatorname{Spec} R, R_{p}$ has finite Krull dimension. By the previous theorem, $M_{p}$ has a flat cover as an $R_{p}$-module. Then it is not very difficult to check that this cover is a flat precover as $R$-modules. Therefore, $M_{p}$ has a flat cover as an $R$-module by Theorem 3.1 of [4].

We remark that since all local rings and coordinate rings of affine algebraic varieties have finite Krull dimension, every module over such rings has a flat cover. But we still do not know if this holds for any commutative Noetherian ring. 


\section{ACKNOWLEDGMENT}

The author expresses his gratitude to Professor Edgar Enochs for his constant encouragement and to the referee for his useful comments on this paper.

\section{REFERENCES}

1. M. Auslander and I. Reiten, Applications of contraviariantly finite subcategories, Adv. Math. 86 (1991), 111-152.

2. H. Bass, Finitistic dimension and a homological generalization of semiprimary rings, Trans. Amer. Math. Soc. 95 (1960), 466-488.

3. R. Belshoff, E. Enochs, and J. Xu, The existence of flat covers, Proc. Amer. Math. Soc. (to appear).

4. E. Enochs, Injective and flat covers, envelopes and resolvents, Israel J. Math. 39 (1981), 189-209.

5. __, Torsion free covering modules, Proc. Amer. Math. Soc 14 (1963), 884-889.

6. __ Flat covers and flat cotorsion modules, Proc. Amer. Math. Soc. 92 (1984), 179-184.

7. __ Covers by flat modules and submodules of flat modules, J. Pure Appl. Algebra 5 (1989), 33-38.

8. L. Gruson and C. U. Jensen, Dimensions cohomolgiques relieés aux foncteurs $\underbrace{}_{\text {lim }}$, Lecture Notes in Math., vol. 867, Springer-Verlag, New York, pp. 234-294.

9. M. Raynaud and L. Gruson, Criteres de platitude et de projectivite, Invent. Math. 13 (1971), 303-310.

10. B. Stenstrom, Rings of quotients: An introduction to methods of ring theory, Springer-Verlag, New York, 1975.

Department of Mathematics, University of Kentucky, Lexington, Kentucky 405060027

E-mail address: abc@ms.uky.edu 